\title{
Targeted positioning of quantum dots inside 3D silicon photonic crystals revealed by synchrotron $\mathrm{X}$-ray fluorescence tomography
}

\author{
Andreas S. Schulz, ${ }^{\dagger,}$ Cornelis A. M. Harteveld, ${ }^{\dagger}$ G. Julius Vancso, \\ Jurriaan Huskens, ${ }^{\ddagger}$ Peter Cloetens, ${ }^{*, \S}$ and Willem L. Vos ${ }^{*, \dagger}$ \\ †Complex Photonic Systems (COPS), MESA+ Institute for Nanotechnology, University of \\ Twente, P.O. Box 217, 7500 AE Enschede, The Netherlands \\ $\ddagger$ Molecular Nanofabrication (MNF), MESA+ Institute for Nanotechnology, University of \\ Twente, P.O. Box 217, 7500 AE Enschede, The Netherlands \\ \Materials Science and Technology of Polymers (MTP), MESA+ Institute for \\ Nanotechnology, University of Twente, P.O. Box 217, 7500 AE Enschede, The Netherlands \\ $\S E S R F-T h e$ European Synchrotron, CS40220, 38043 Grenoble, France \\ E-mail: cloetens@esrf.eu; w.I.vos@utwente.nl
}

\section{Abstract}

It is a major outstanding goal in nanotechnology to precisely position functional nanoparticles, such as quantum dots, inside a threedimensional (3D) nanostructure in order to realize novel functions. Once the 3D positioning is performed, the challenge arises how to nondestructively verify where the nanoparticles reside in the 3D nanostructure. Here, we study 3D photonic band gap crystals made of Si that are infiltrated with PbS nanocrystal quantum dots. The nanocrystals are covalently bonded to polymer brush layers that are grafted to the Si-air interfaces inside the 3D nanostructure using surface-initiated atom transfer radical polymerization (SI-ATRP). The functionalized 3D nanostructures are probed by synchrotron $\mathrm{X}$ ray fluorescence (SXRF) tomography that is performed at $17 \mathrm{keV}$ photon energy to obtain large penetration depths and efficient excitation of the elements of interest. Spatial projection maps were obtained followed by tomographic reconstruction to obtain the $3 \mathrm{D}$ atom density distribution with $50 \mathrm{~nm}$ voxel size for all chemical elements probed: $\mathrm{Cl}, \mathrm{Cr}, \mathrm{Cu}, \mathrm{Ga}, \mathrm{Br}, \mathrm{Pb}$.
The quantum dots are found to be positioned inside the 3D nanostructure, and their positions correlate with the positions of elements characteristic of the polymer brush layer and the ATRP initiator. We conclude that X-ray fluorescence tomography is very well suited to non-destructively characterize 3D nanomaterials with photonic and other functionalities.

\section{Keywords}

3D integration, complementary metal-oxidesemiconductor (CMOS), nanofabrication, photonic crystals, quantum dots, $\mathrm{x}$-ray fluorescence imaging

\section{Introduction}

Three-dimensional (3D) functionalized nanostructures are drawing fast-growing attention for their advanced applications in nanophotonics, ${ }^{[1] 3]}$ photovoltaics, $\frac{45]}{15}$ capacitors in electronics, ${ }^{6}$ gas sensing, ${ }^{7}$ materials for electrochemical energy conversion and storage, ${ }^{[8}$ and bat- 
teries. $\sqrt{9}$ The functionalization of these nanostructures is the result of the infiltration of active nanoparticles, e.g., fluorophores in nanophotonic light sources, $13-15$ antibodies for biochemical sensors of diseases, or quantum dots for photovoltaics. $\frac{16 \mid 17}{1 n}$ most cases, the performance of the 3D functionalized nanostructure depends on the precise positioning of the nanoparticles inside the 3D nanostructure, with nanometer precision. In nanophotonics, for instance, fluorescing nanoparticles should reside at positions where the local density of optical states is either maximal, in case of cavities or antennae, 18 or minimal in case of a photonic band gap. $21 / 22$ In biochemical detectors, antibodies should be positioned on the internal interfaces of the 3D substrate for maximum reactivity and selectivity. 23

In this representative study, we fabricate 3D photonic band gap crystals from silicon with the diamond-like inverse woodpile structure consisting of two perpendicular arrays of interpenetrating nanopores, $\stackrel{24}{24}$ see figure 1(a). We infiltrate functional quantum dot nanoparticles in these crystals that are precisely positioned relative to the silicon-air interfaces by means of polymer brushes 2526 that grow from initiator molecules placed on the internal silicon interfaces, see figure 1(b). In previous photonic band gap crystal studies, quantum dots were randomly positioned in the crystal, $\frac{3}{3}$ whereas brushes will allow to selectively position the dots at places where they experience the maximal band gap effects. ${ }^{22}$ Once the nanoparticles have been infiltrated, the challenge addressed here is to find back their final positions. Therefore, the first requirement is to find a probing method with the first requirement that it provides local information deep inside a 3D nanostructure with nanometer spatial resolution. A second requirement is that the method is element specific to verify that the infiltrated nanocrystals are located in the desired positions and to characterize the infiltration mechanism or process. A third major requirement is that the probing technique must be non-destructive, hence the nanomaterial remains functional and ready for further integration after the inspection. While the widely used scanning elec-

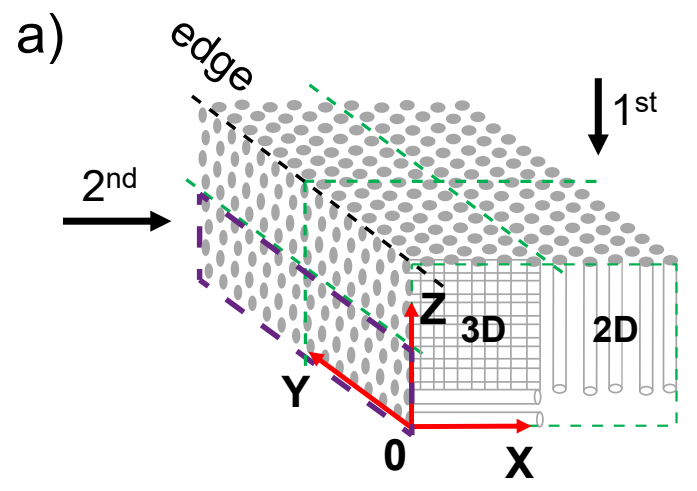

b)

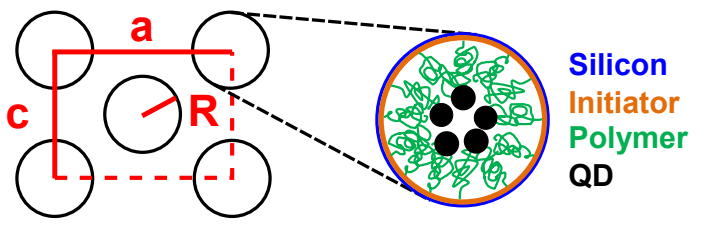

c)

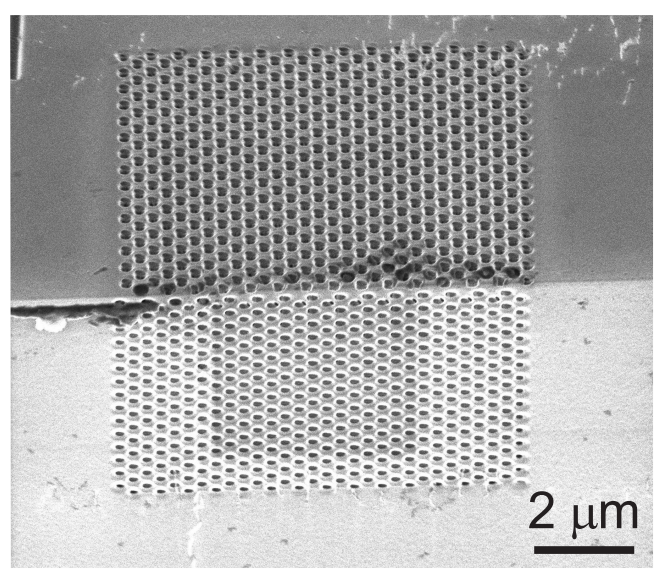

Figure 1: 3D photonic crystal and chemical positioning of quantum dots inside of the nanostructure. (a) Schematic of an inverse woodpile photonic crystal on the edge of a silicon beam. Green dashed lines indicate the two surfaces into which nanopores are etched. The 3D and 2D parts of the crystal are indicated in the XZ front face. (b) View along the pores showing the lattice parameters $a, c$ (with $a=\sqrt{2} c$ ) and pore radius $\mathrm{R}$. Zoomed-in cross-section of one pore with targeted surface-chemistry: ATRP initiator layer (orange), polymer chains forming brushes (green) and covalently attached $\mathrm{PbS}$ quantum dots (red) on top of silicon (black). (c) Scanning electron microscope (SEM) image of a $3 \mathrm{D}$ photonic crystal viewed from $45^{\circ}$ on the edge of the silicon beam showing the XY (top) and the XZ surfaces (bottom); the scale bar indicates $2 \mu \mathrm{m}$.

tron microscopy (SEM) non-destructively re- 
veals the periodically ordered external surfaces of the photonic crystal (figure 1(c)), internal nanoparticles are hidden, even at a much higher resolution.

A common approach in nanotechnology to view elements inside $3 \mathrm{D}$ nanostructures is to cleave or ion-mill the sample and then probe the exposed surface with energy dispersive Xray spectroscopy, 27 time-of-flight secondaryion mass spectrometry, ${ }^{28} \mathrm{X}$-ray photoelectron spectroscopy ${ }^{29}$ or Fourier-transform infrared or Raman spectroscopy. 30 A major disadvantage of this approach is that it is destructive. Moreover, several of the probing methods have coarse spatial resolutions in the range of $10 \mu \mathrm{m}$ or even coarser. X-ray techniques offer suitable non-destructive analysis methods due to their high penetration and high resolution. 31132 Anomalous small-angle scattering of X-rays allows to obtain contrast variation in a multicomponent system. $\stackrel{3334}{ }$ Unfortunately, the number of elements that can be distinguished is limited because many incident X-ray wavelengths have to be independently tuned, and it is very difficult to obtain local information from such a scattering method. Here, we demonstrate the use of X-ray fluorescence tomography using a synchrotron source,, 35 that meets all above requirements, to inspect designed functional nanostructured samples.

In X-ray fluorescence tomography, the sample is illuminated with a finely focused X-ray beam to excite X-ray fluorescence that is characteristic for each element in the sample, see figure 2(a). At $\theta=-47^{\circ}$ the incident beam passes obliquely through the crystal. The right detector will detect the fluorescence signal that is integrated along the incident X-ray beam (the left detector will detect little signal as it is attenuated by the substrate.) At $\theta=-2^{\circ}$ the incident beam traverses the whole $500 \mu \mathrm{m}$-thick Si substrate, which is feasible on account of the high photon energy of $17 \mathrm{keV}$. The detected fluorescence signal is integrated along the $\mathrm{X}$ direction, hence the left detector effectively sees a projection of the object in the YZ-plane. At $\theta=43^{\circ}$ the incident beam first passes through even more substrate thickness (by projection) before entering the photonic crystal. Both de- a)
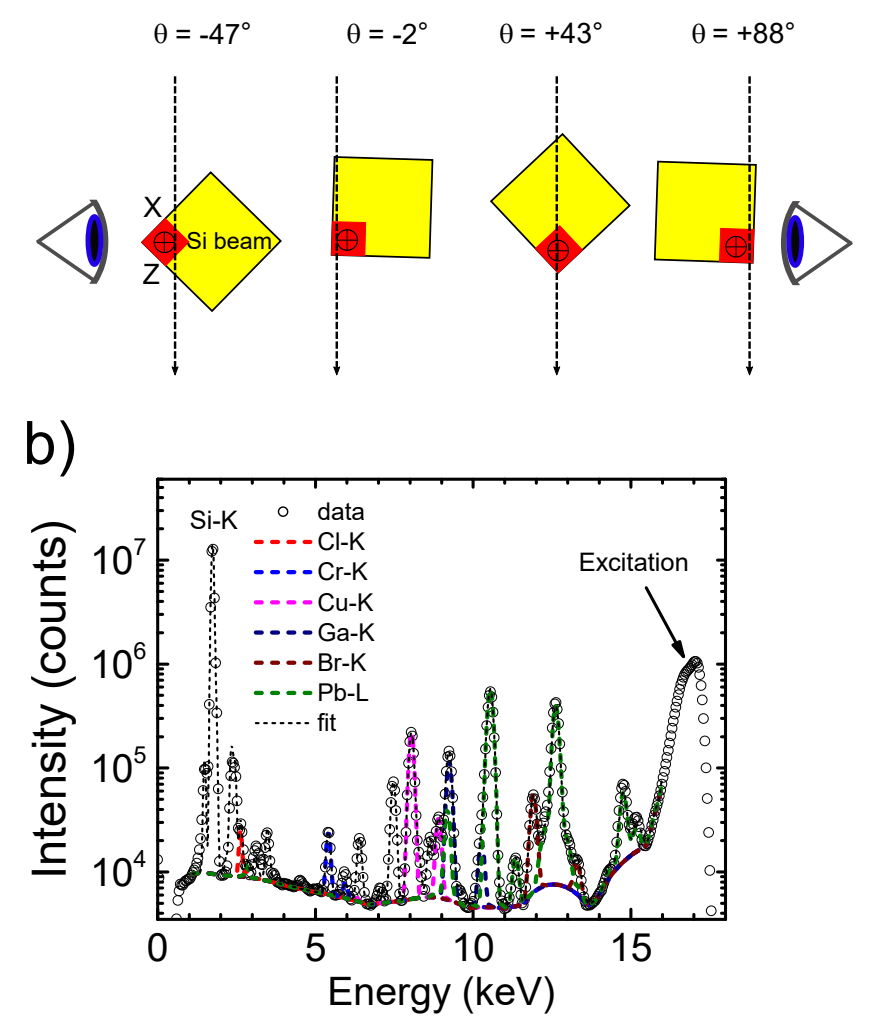

Figure 2: Sample mounting and X-ray fluorescence spectra. (a) Top view showing how the X-ray beam traverses the sample on the Si substrate at four different angles $\theta$. Two detectors collect X-ray fluorescence signal on the left and right sides. (b) X-ray fluorescence spectrum $I(E, \theta)$ (circles) of one of the crystals averaged over all $240 \times 240$ pixels in the image at $\theta=-47^{\circ}$. The analyzed $\mathrm{Cl}, \mathrm{Cr}, \mathrm{Cu}, \mathrm{Ga}, \mathrm{Br}$, $\mathrm{Pb}$ transitions are shown in order of increasing photon energy.

tectors see an oblique projection of the sample. At $\theta=88^{\circ}$ the X-ray beam travels nearly in the high-symmetry -Z-direction, the (left) detector detects fluorescence integrated along the -Z-direction and thus effectively detects a projection of the object in the XY-plane.

Figure2(b) shows a typical X-ray fluorescence spectrum that is averaged over all $240 \times 240$ pixels. We identify the involved elements from their known X-ray emission with constraints from the known chemical compounds involved in the sample preparation (see Methods). The peak at $1.74 \mathrm{keV}$ is the $\mathrm{Si}-\mathrm{K}_{\alpha}$ transition from the photonic crystal matrix. In order of increas- 
ing photon energy, chlorine, chromium, copper, gallium, bromine, and lead are detected. Chlorine features in the $\mathrm{Cu}(\mathrm{I}) \mathrm{Cl}$ species used as catalyst in the polymerization and in the termination group of the polymer brushes (see figure $1(\mathrm{~b}))$. Chromium appears in the sample as the hard-mask material for the deep reactiveion etching of the pores that define the 3D photonic crystal. Copper is introduced by the two catalyst species $\mathrm{Cu}(\mathrm{I}) \mathrm{Cl}$ and $\mathrm{Cu}(\mathrm{II}) \mathrm{Br}$ that are incompletely washed out due to the limited permeability of such a complex 3D porous nanostructure. Gallium occurs since it is used to write the etch mask by means of focused-ion beam milling. Bromine features in the ATRPinitiator monolayer for the brush synthesis and also in the $\mathrm{Cu}(\mathrm{II})$ catalyst species used in the polymerization (see figure 1(b)). Finally, lead is the signature of the infiltrated $\mathrm{PbS}$ quantum dots with a strong signal since it is a heavy element. We note that sulfur from the quantum dots is not detected, since the signal is attenuated due to the low photon energy and is likely overwhelmed by the strong $\mathrm{Si}_{\alpha}$ peak at 1.74 $\mathrm{keV}$. The peak at $17 \mathrm{keV}$ is the excitation by the incident X-ray beam that is broadened due to Compton scattering and a finite detector resolution.

At every sample orientation $\theta$ (figure 2(a)), the X-ray focus is raster scanned through the crystal (see Methods). The X-ray fluorescence signals separated for all elements yield so-called projection maps. These are 2D maps of the elemental number density per pixel area integrated along the X-ray beam while it traverses the sample. Figure 3 shows examples for Ga and $\mathrm{Br}$ at two different orientations $\theta$.

Figure 3(a) shows the gallium signal projected on the YZ plane. From left to right, we see (faintly) the nanopore entrances (on the YZ crystal surface) that are defined in the lithography step by focused-ion beam milling using $\mathrm{Ga}$ ions. At two thirds from the left, there is a bright streak of Ga-signal in the Y-direction, because the fluorescence signal is added (projected) in the X-direction for all nanopore entrances in the XY crystal surface.

Figure 3(b) shows the gallium signal projected on the XY plane. At one fourth from

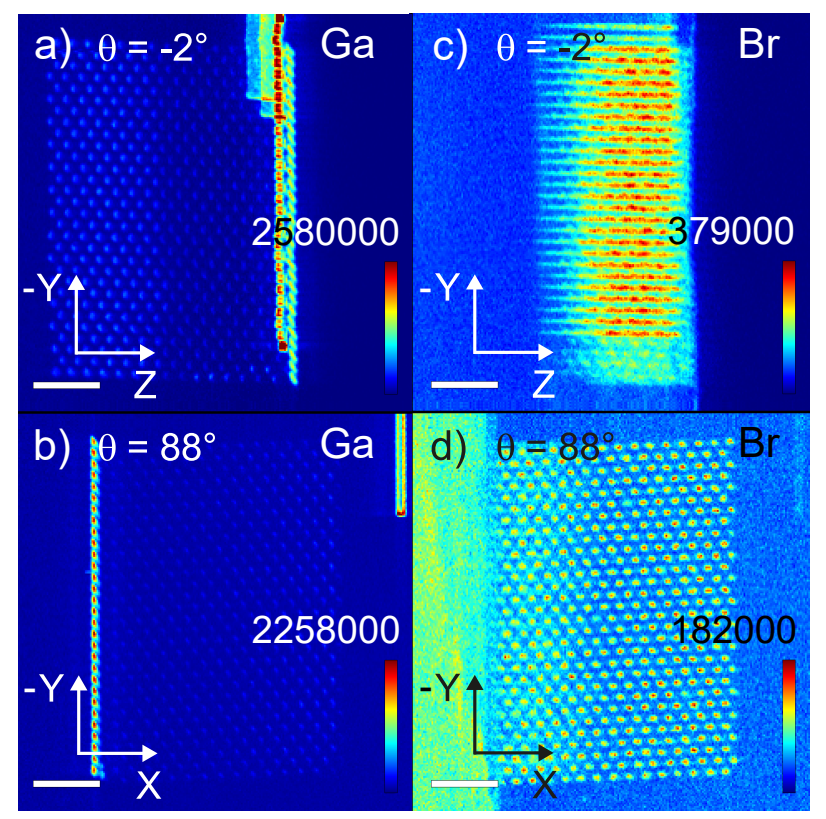

Figure 3: Projection maps of the number of Ga and $\mathrm{Br}$ atoms per pixel (with area $50 \times 50 \mathrm{~nm}^{2}$ ), integrated along the propagation direction of the beam, for sample orientations: a) Ga at $\theta=-2^{\circ}$, b) Ga at $\theta=88^{\circ}$, c) Br at $\theta=-2^{\circ}$, d) $\mathrm{Br}$ at $\theta=88^{\circ}$. Color bars with the density scales are given in each panel.

the left, there is a bright streak of Ga-signal in the Y-direction, because the fluorescence signal is added (projected) in the Z-direction for all nanopore entrances in the YZ crystal surface. Further to the right, we (faintly) see the nanopore entrances on the XY crystal surface. These observations confirm that the gallium atoms are effective markers of the external crystal surface.

In Figure 3(c) we observe the Br density in the photonic crystal from a high-symmetry angle viewing along the $\mathrm{X}$-axis onto the $\mathrm{YZ}$ plane. The $\mathrm{Br}$ density is arranged in horizontal stripes, due to the Z-directed pores. Therefore, we conclude that $\mathrm{Br}$ is present throughout the whole extent of the nanopores. Thus, the ATRPinitiator has infiltrated the pores of the 3D silicon photonic crystal to form a monolayer throughout the whole structure (based on previous results on flat $\mathrm{Si}$ wafers $\left.{ }^{26}\right)$, which is crucial for further functionalization of the surface inside the 3D silicon crystal pores. It is also conceivable that part of the $\mathrm{Br}$ density is caused by incompletely rinsed $\mathrm{Cu}(\mathrm{II}) \mathrm{Br}$ catalyst that 
is used in the synthesis. In both cases, we conclude that intended chemistry has taken place inside the nanopores.

In Figure 3(d) we observe the projection in the XY plane that shows the periodicity of the nanopores as individual disks of $\mathrm{Br}$ atoms. We do not observe thin circular shapes as expected in case of purely surface deposition on perfectly cylindrical pores. Since the pores are not perfectly perpendicular to the XY-plane and have a depth-dependent diameter (known as tapering $\left.{ }^{38}\right)$, the projected $\mathrm{Br}$ density appears as filled ellipses. On the left side the edge of the silicon beam is visible.

Projection maps are collected while rotating the sample by $180^{\circ}$, see figure 2(a). After tomographic reconstruction (see Methods), one obtains a position-resolved 3D density map of all elements that are detected inside the nanostructure. In case of $\mathrm{Cl}$ and $\mathrm{Cr}$, the signals were relatively weak (only a few times $10^{4}$ counts after background subtraction) so that their projection maps were relatively noisy, hence no firm conclusions could be drawn on the positions of these elements.

Figure 4(a,b) show XZ cross-sections of the $3 \mathrm{D}$ density map of gallium $N_{v, G a}(\mathrm{X}, \mathrm{Y}, \mathrm{Z})$. The gallium is located near the external surfaces of the photonic crystal, which is reasonable since Ga-ions are used to define the etch masks for the nanopores on the external surfaces of the $\mathrm{Si}$ beam substrate by focused-ion beam milling. 39 Since there is hardly any Ga-signal from the bulk of the Si substrate, there are few artifacts due to the tomographic image reconstruction, which confirms that the reconstruction has converged well.

Figures 4(c,d) show XZ cross-sections of the 3D density map of bromine atoms $N_{v, B r}$ $(\mathrm{X}, \mathrm{Y}, \mathrm{Z})$, see Figure 1(b). The $\mathrm{Br}$ density is distributed in vertical stripes in the Z-direction, showing that the $\mathrm{Br}$ atoms have infiltrated the nanopores, to as deep as $\Delta \mathrm{Z}=3.5 \mu \mathrm{m}$. All Z-directed nanopores are filled from the sample surface, since all pores over an X-extent $\Delta \mathrm{X}=7.2 \mu \mathrm{m}$ reveal similar $\mathrm{Br}$ density profiles. We also observe that Br-atoms are infiltrated in the $\mathrm{X}$-directed pores at intermediate Y-coordinates (see Supplementary movie

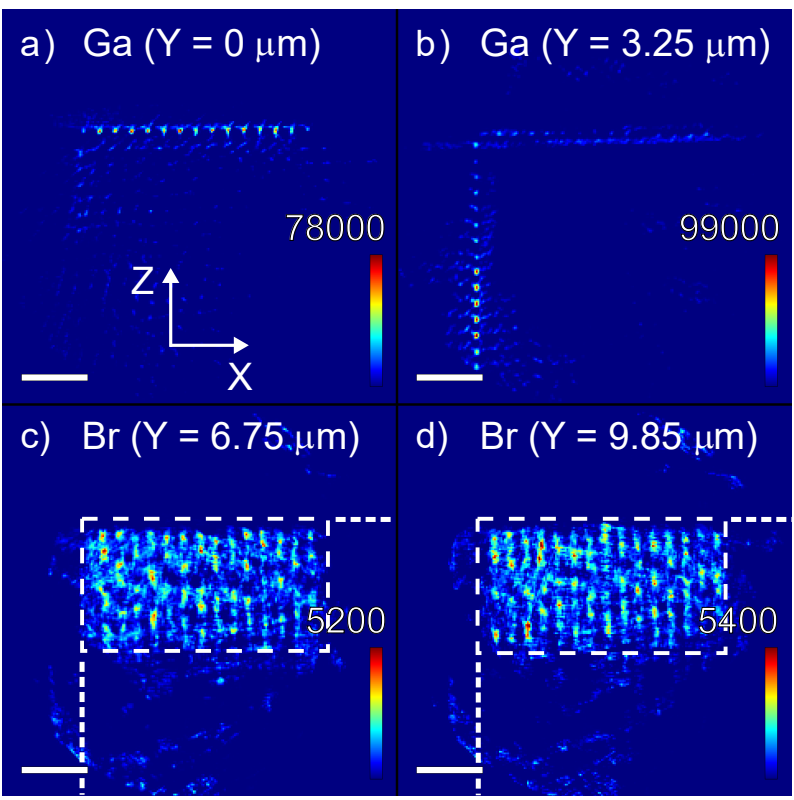

e)

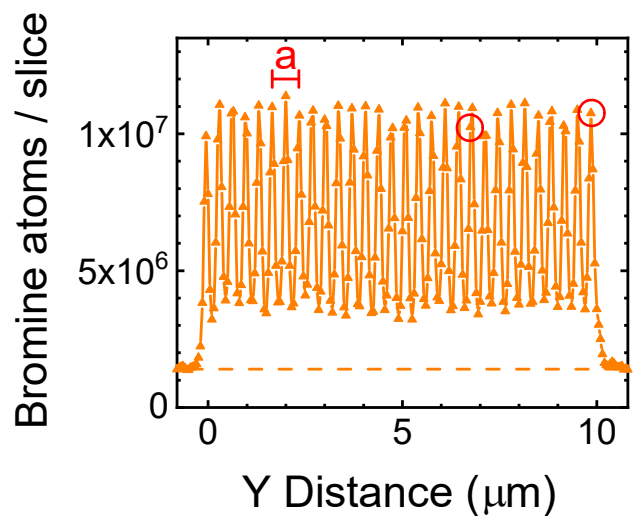

Figure 4: $\mathrm{XZ}$ cross-sections of the Ga atomic density at depths $\mathrm{Y}=0 \mu \mathrm{m}$ (a) and $\mathrm{Y}=$ $3.25 \mu \mathrm{m}$ (b). XZ cross-sections of the Br atomic density at $\mathrm{Y}=6.75 \mu \mathrm{m}(\mathrm{c})$ and $\mathrm{Y}=9.85 \mu \mathrm{m}$ (d). The scale bars represent $2 \mu \mathrm{m}$, the intensity bars the number of atoms per voxel (with volume $50^{3} \mathrm{~nm}^{3}$ ) and white dashed lines delineate the crystal's cross section and the external surface. (e) Number of bromine atoms per slice integrated along $Z$, as a function of $\mathrm{Y}$. The lattice parameter $a$ of the photonic crystal is shown. The periodicity of the pores is clearly visible. Red circles indicate the $\mathrm{Y}$-coordinates in c) and d).

S2). Thus, the Br atoms effectively outline the nanopore structure in the silicon. These observations match our expectations because the initiator molecules were introduced as a vapor that readily diffuses into a porous sample. Therefore, the silane monolayer due to the ATRP de- 
position method resides on the surfaces of the nanopores. The presence and homogeneity of the silane monolayer influences and defines the subsequent functionalization with the polymer brush layer.

Figure 4(e) shows the total number of $\mathrm{Br}$ atoms per XZ slice as a function of $Y$-position. We observe that the bromine density shows a sine-like profile in the whole crystal between $\mathrm{Y}=0$ to $10 \mu \mathrm{m}$. The period is equal to $(a / 2)$ which is sensible because fluorescent signal from inside the pores is observed twice per unit cell since there are two planes of pores within each unit cell, as shown in Figure 1(b). We observe that the maximum amplitude is constant to within $13 \%$ and the minimum amplitude is constant to within $21 \%$, confirming a fairly homogeneous infiltration. The open red circles mark the Y-positions of the slices in Figures $4(\mathrm{c}, \mathrm{d})$. We therefore conclude that the upper amplitude represents the bromine densities in the pores in $Z$-direction, whereas the lower amplitude represents the bromine densities in the pores in the X-direction. The amplitude oscillates between $3.6 \times 10^{6}$ and $0.8 \times 10^{7}$ bromine atoms per slice, whereas the finite density outside the crystal is $1.3 \times 10^{6}$ bromine atoms per slice. When we correct the densities in the crystal for this background, the density in the pores in the Z-direction equals $\left(9.4 \times 10^{6} / 2.2 \times 10^{6}\right)=4.3 \times$ greater than in the pores in the $\mathrm{X}$-direction. One possible explanation for the different average $\mathrm{Br}$ densities is that the pores in the $\mathrm{X}$-direction were etched in the second etching step with the presence of fluorocarbons on the YZ surface. It is then conceivable that these fluorocarbons that were used in the protection steps of the etching process were incompletely removed by the oxygen plasma cleaning in the etching machine after the etching of the first set of pores in the Zdirection, thereby (partly) blocking the infiltration of these nanopores.

Figure 5(a,b) show XZ cross-sections of the $3 \mathrm{D}$ density map of lead atoms $N_{v, P b}$ (X, Y, Z), which are the cations of the infiltrated $\mathrm{PbS}$ quantum dot nanoparticles, see figure 1(b). The $\mathrm{Pb}$ density extends along the pores in the Z-direction, starting from the XY top surface
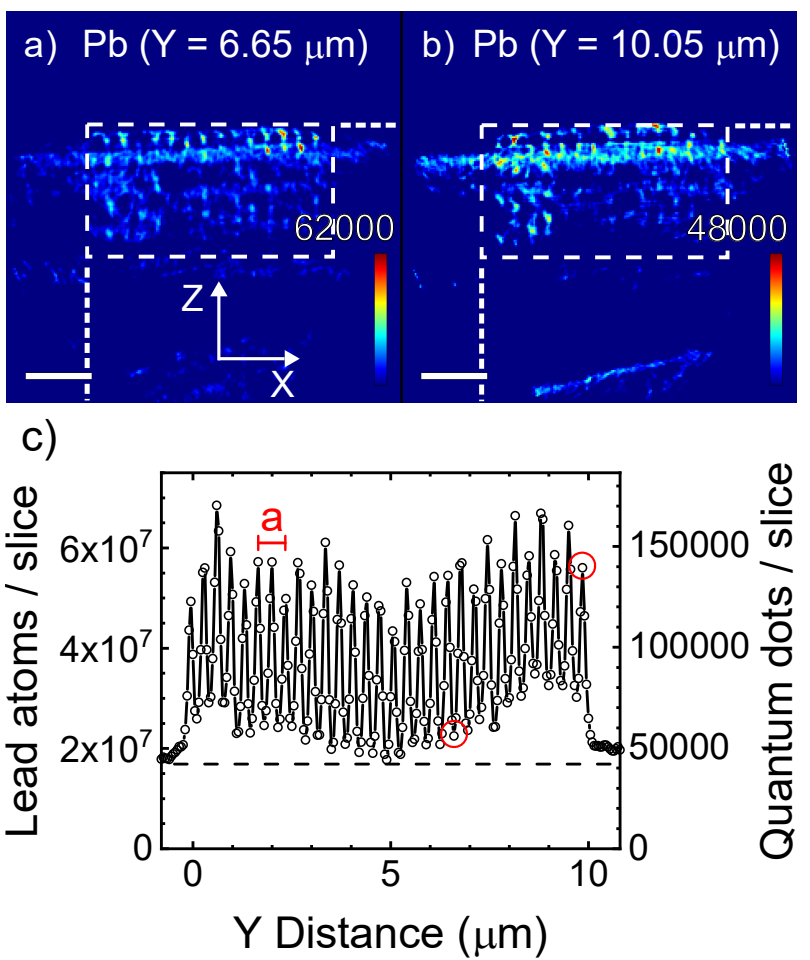

Figure 5: (a, b): XZ cross-sections of the reconstructed sample volume for the $\mathrm{Pb}$ atoms at depths $\mathrm{Y}=6.65 \mu \mathrm{m}(\mathrm{a}), \mathrm{Y}=10.05 \mu \mathrm{m}$ (b). The scale bar represents $2 \mu \mathrm{m}$, the intensity bar the number of lead atoms per voxel and white dashed lines delineate the crystal's crosssection. c) Density of lead atoms per XZ-slice versus $Y$, the right ordinate shows the corresponding number of quantum dots (taking 402 $\mathrm{Pb}$ atoms per dot). The lattice parameter $a$ of our photonic crystal is indicated. The periodicity of the pores is clearly visible. The red circles show the Y-coordinates in (a) and (b).

and extending about $3.5 \mu \mathrm{m}$ into the crystal. We conclude that the quantum dots have indeed been successfully infiltrated into the nanopores of the $3 \mathrm{D}$ photonic crystal.

To characterize in more detail the infiltration of the quantum dots in each nanopore, we plot in figure 5(c) the density of lead atoms integrated along $\mathrm{Z}$ as a function of $\mathrm{Y}$-coordinate. The $\mathrm{Pb}$ density oscillates throughout the whole crystal between $\mathrm{Y}=0$ to $10 \mu \mathrm{m}$ with a period equal to $(\mathrm{a} / 2)$, as with the $\mathrm{Br}$ data in figure 4(e), due to two planes of nanopores per unit cell. The $\mathrm{Pb}$ density amplitude in Figure 5(c) oscillates between $1.8 \times 10^{7}$ and $6.8 \times 10^{7}$ lead atoms per slice. Outside the crystal, the 
lead density is between $1.8 \times 10^{7}$ and $2.1 \times 10^{7}$ atoms/(50 $\mathrm{nm} \times 50 \mathrm{~nm} \times 3.5 \mu \mathrm{m})$ which is mostly determined by quantum dots on the external sample surface (a real signal) and by artifacts due to the 2D structure that lies behind the $3 \mathrm{D}$ structure. At $\mathrm{Y}=5 \mu \mathrm{m}$ the $\mathrm{Pb}$ density modulation is somewhat reduced before increasing again towards the right edge of the crystal at $\mathrm{Y}=10 \mu \mathrm{m}$.

Since we know from the Br density (see figure $4(\mathrm{c}, \mathrm{d}))$ that the Z-directed nanopores have a depth of $3.5 \mu \mathrm{m}$, the exhilarating conclusion arises that the quantum dots have been successfully infiltrated over the full depth of the nanopores. Similar to $\mathrm{Br}$ at other Ycoordinates (see Supplementary movie S3), we also observe $\mathrm{Pb}$ over the full depth of the $\mathrm{X}$ directed pores. Therefore, our strategy to infiltrate quantum dots inside 3D nanostructures by attaching them to polymer brushes has been successful. Moreover, this result demonstrates that X-ray fluorescence tomography is a powerful method to characterize the infiltration of functional nanoparticles in complex 3D architectures.

We observe that the maximum amplitude in Figure 5(c) is constant to within 37\% and the minimum amplitude is constant within $50 \%$. These amplitudes for the lead atoms are less homogeneous than for bromine, which is reasonable for three reasons. (i) The $\mathrm{Br}$ atoms originating from the initiator molecules are introduced in the vapor phase, hence the diffusion is the fastest, leading to the most homogeneous infiltration. Moreover, the rinsing of excess molecules of this species also likely has the most homogeneous distribution. (ii) The bromine atoms originating from the $\mathrm{Cu}(\mathrm{II}) \mathrm{Br}$ catalyst occur in the polymerization solution. These bromine atoms diffuse less readily in the nanopores than the bromine atoms (in the initiator) introduced via the vapor. (iii) Since the quantum dots that are introduced as nanoparticles in suspension are much larger than the initiator and the catalysts molecules, it is a reasonable inference that the QDs diffuse even less readily than the vapor and the polymerization solution, thereby leading to a greater inhomogeneity. Moreover, additional inhomogeneity arises during the rinsing of excess of quantum dots after infiltration. Based on the details explained above, we expect that the maximum and the minimum amplitudes of the lead signal vary more than for bromine.

Let us evaluate our results in light of the three main requirements that we set out with. Firstly, we aimed for (a) nanometer resolution (b) at great depths inside 3D nanostructures. (a) At present, the resolution is determined by the size of the X-ray focus (23 nm by $37 \mathrm{~nm}$, see Methods) and the step size of the spatial scan $(50 \mathrm{~nm})$, where the latter was in turn set by the total measurement duration. It would be advantageous to further improve the resolution as it would allow to resolve the brush thickness. A straightforward way to improve the resolution such that it is limited only by the focus size, is to increase the incident photon flux and improve the detector technology. This is exactly what is being done in the ongoing refurbishments of several large synchrotrons including ESRF. The increased coherence of the new synchrotron X-ray beams will also allow for employing smaller foci and hence a further improved resolution. (b) To probe elements deep inside nanostructures, we employed high-energy X-ray photons $(17 \mathrm{keV})$ to achieve a high penetration depth. Such a high photon energy also allows for samples to be integrated on thick substrates like wafers. ${ }^{40}$ At the ID16A instrument used in the present study, it is also feasible to excite at an even higher photon energy of 33.5 $\mathrm{keV}$, but this makes little sense since a major limitation is the absorption of the emitted Xray fluorescence that has a fixed energy. Exciting at higher photon energy will reduce the excitation efficiency and therefore also reduce the sensitivity. Our choice of $17 \mathrm{keV}$ is nearly ideal to excite all elements up to $\mathrm{Br}$ (absorption edge at $13.5 \mathrm{keV}$ ) and $\mathrm{Pb}$ (highest L-edge at $15.9 \mathrm{keV}$ ). The $\mathrm{K}$-edge of $\mathrm{Pb}$ at $88 \mathrm{keV}$ is out of reach with nano-focusing, moreover, exciting this absorption edge would result in highly inefficient excitation of other, lighter, elements.

Secondly, we aimed for element specific detection. Indeed, we successfully detected $\mathrm{Br}$, $\mathrm{Ga}, \mathrm{Pb}$ and $\mathrm{Cr}$ with a high signal to noise ratio. For $\mathrm{Cl}$ and $\mathrm{Cu}$ the collected data had a low 
signal to noise ratio, due to a surface contamination that was hiding the weaker bulk signal. Moreover, the signal of these low-Z elements at relatively low photon energy is susceptible to self-absorption by the bulky sample. The outcome could potentially be improved for these low-Z elements by using a confocal detection scheme ${ }^{41}$ or wavelength dispersive X-ray spectroscopy. $\stackrel{42}{~ N e v e r t h e l e s s, ~ c o m b i n i n g ~ t h e s e ~ t e c h-~}$ niques with nano-tomography remains a major technical challenge. It is an interesting suggestion to design the monomer for the polymer synthesis with a heavier element as a marker, such that it is more readily detected by X-ray fluorescence.

Thirdly, we aimed for a non-destructive probing method. Our method meets this requirement as no irreversible mechanical modifications like cleaving or milling are needed. To further confirm the integrity of our samples, we verified that we could still detect near infrared luminescence from the $\mathrm{PbS}$ quantum dots after the X-ray experiments.

In summary, we have performed X-ray fluorescence tomography to locate the positions of quantum dots that were infiltrated inside a 3D nanostructure, which was made from silicon. This demonstration opens the road to various applications. In nanophotonics it is known that the fundamental local density of optical states (that controls the fluorescence of embedded emitters) has interesting properties, either maxima and minima, that do not necessarily appear at interfaces between constituent material. ${ }^{20}$ Thus, to optimally control an emitter, one has to position the emitter at these positions. Assuming that the technology to position the emitter exists (see, e.g., Ref. ${ }^{26}$ ), one must then probe the resulting positions to verify the functionality of the device, and this is exactly the step forward offered by X-ray fluorescence tomography in our work. In other technologies, like batteries or sensors, it is vital to position functional nanoparticles on the interfaces between constituent materials or phases, while the functionality or its efficiency greatly decrease if the nanoparticles are displaced. So also for these applications, X-ray fluorescence tomography can verify the intended positions to a high (nanometer) precision, and thereby greatly contribute to improved batteries and sensors, see e.g., Ref. $\underline{43}$

\section{Methods Section}

\section{D Si Photonic Crystals}

Our 3D photonic band gap crystals were fabricated by a CMOS-compatible fabrication process that is described in detail in Refs. $\frac{38 / 39 / 44}{\text { As }}$ substrates, we employ Si beams (cross-sections $0.5 \times 0.5 \mathrm{~mm}^{2}$ ) that are chemically etched to obtain perpendicular crystal surfaces. A thin $50 \mathrm{~nm}$ Cr layer serves as a hard etch mask that is deposited on two adjacent surfaces of a beam. In the Cr layer, we define an etch mask in a single step on both faces of the beam using focused-ion beam writing with Ga ions. 39 We perform deep reactive ion etching using the Bosch process (a time-multiplexed alternating process that alternates etching and lamination steps) to etch two perpendicular arrays of deep nanopores. $\frac{38}{1 n}$ the etching step, pores are etched with $\mathrm{SF}_{6}$ and in the lamination step the pore walls are protected with a CF polymer layer. Figure 1 shows a SEM image of a successfully etched photonic crystal. The overlap region of the pores entering into the XY surface (top) and into the XZ surface (bottom) mutually cross inside the beam and form the $3 \mathrm{D}$ cubic inverse woodpile structure that has a broad photonic band gap. 24/45

The size and shape of the resulting 3D cubic inverse woodpile structure depends on the etch depths of the both sets of pores. The deeper each set of pores is etched into the silicon, the larger the volume of the inverse woodpile structure. For this specific sample studied here, the resulting 3D volume was smaller than aimed for. The reason is that the second etching step resulted in shorter pores than the first one etched due to high amounts of fluorocarbons on the surface for the second set of pores. Therefore, the pores in the X-direction do not cross all pores etched in the Z-direction (see Fig. 1(a)), therefore, at larger X-positions 
there is a $2 \mathrm{D}$ crystal of Z-directed pores adjacent to the 3D crystal structure near the edge of the wafer.

\section{Cleaning}

The Cr hard mask was removed in ceric ammonium nitrate-based etchant for $60 \mathrm{~s}$. The beam was intensively rinsed for $1 \mathrm{~h}$ with deionized (DI) water and dried in a nitrogen stream.

RCA-2 cleaning ${ }^{1}$ was used to remove metal residues from the samples. $\stackrel{46}{\mathrm{~A}}$ glass beaker was placed on a hot plate, $1500 \mathrm{~mL}$ DI water was added, and while magnetically stirring, $300 \mathrm{~mL}$ of hydrochloric acid was slowly added. The solution was heated to $70^{\circ} \mathrm{C}, 300 \mathrm{~mL}$ hydrogen peroxide was slowly added under stirring. Once the solution reached $70^{\circ} \mathrm{C}$ the samples were added and were kept in the RCA-2 solution for $15 \mathrm{~min}$. The samples were repeatedly rinsed in a water bath until a resistivity $>10$ $\mathrm{M} \Omega \mathrm{cm}$ was reached, indicative of an acid-free environment.

Organic traces were removed by placing a sample in a bath of $\mathrm{HNO}_{3}(99 \%$, room temperature) for $10 \mathrm{~min}$. The sample was transferred to a second bath under the same conditions and cleaned for another $10 \mathrm{~min}$, and rinsed with DI water and dried under a nitrogen flow. The dried sample was transferred into a closed beaker of boiling $\mathrm{HNO}_{3}$ (69\% in water, $95^{\circ} \mathrm{C}$ ) and cleaned for $10 \mathrm{~min}$. The sample was rinsed with DI water and dried under nitrogen. Samples were placed in a clean beaker that was closed and kept on a hot plate to prevent water to enter the pores before the furnace cleaning. The samples were thermally treated in a furnace at $800^{\circ} \mathrm{C}$ under $100 \% \mathrm{O}_{2}$ for $90 \mathrm{~min}$ to oxidize and remove the remaining fluorocarbons (from the etching step) from the pore surface inside the silicon.

\section{Polymer Brush Infiltration}

The polymer brush infiltration is described ino two parts: firstly, the attachment of the initia-

\footnotetext{
${ }^{1}$ RCA-2 was developed in 1965 by Werner Kern while working for the Radio Corporation of America (RCA).
}

tor to the silicon beam, secondly, the SI-ATRP of the poly(glycidyl methacrylate) brushes.

Chemical vapor deposition of the initiator The ATRP initiator was synthesized following Ramakrishnan et al. $\stackrel{47}{ }$ A piranha solution was prepared with the ratio of 3 parts $\mathrm{H}_{2} \mathrm{SO}_{4}$ to 1 part $\mathrm{H}_{2} \mathrm{O}_{2}$ (volume ratio, v/v\%). The silicon beam was placed into the sample holder. The sample holder was lowered gently into the piranha solution and the sample was cleaned for 30 min. The silicon beam was rinsed 10 times with water and ethanol. A desiccator was rinsed by vacuum pumping for 5 to 10 min. The initiator was taken from a vial using a argon purged syringe. A few droplets of initiator $(30 \mu \mathrm{L})$ were placed inside a small plastic petri dish that was placed in the middle of the desiccator. The Si beam was placed close to the petri dish such that the vapor diffuses into the nanopores of the photonic crystal. The desiccator was vacuum pumped for $15 \mathrm{~min}$, and the chemical vapor deposition (CVD) process proceeded for $16 \mathrm{~h}$. Next, the beam was placed into a flask with $100 \mathrm{~mL}$ toluene in an ultrasonication bath for $20 \mathrm{~s}$ to remove excess initiator. Each substrate was rinsed 10 times with ethanol and water before the substrates were dried under a nitrogen stream. The silicon beam was stored in a nitrogen box.

\section{Chemical synthesis of PGMA polymer} brushes Glycidyl methacrylate (GMA, 10 $\mathrm{mL}, 75 \mathrm{mmol}$ ) monomer and 2,2'-Bipyridine (BiPy, $282 \mathrm{mg}, 1.81 \mathrm{mmol}$ ) were added to a mixture of water $(2 \mathrm{~mL})$ and methanol $(8$ $\mathrm{mL}) . \underline{48}$ The solution was purged with argon for $30 \mathrm{~min}$ under continuous stirring. $\mathrm{CuCl}$ (72.8 mg, $0.74 \mathrm{mmol}$ ) and $\mathrm{CuBr}_{2}$ (7.8 mg, 0.035 mmol) were added to another flask and flushed with argon for $15 \mathrm{~min}$. The monomer solution was transferred by a previously flushed syringe to the catalyst flask. The resulting polymerization solution was stirred for an additional 30 min under argon. The silicon beam was taken out of the nitrogen box shortly before the reaction and was placed into a reaction flask. The reaction flask was attached to a vacuum pump. Afterwards the monomer solution was 
transferred to the reaction flask containing the silicon beam. The polymerization was allowed to proceed for $60 \mathrm{~min}$. The silicon beam was cleaned extensively with acetone and waterfree dimethyl sulfoxide to remove non-reacted monomer, metal complex, and remaining catalyst residues. The silicon beam was dried in a nitrogen stream to obtain the sample with the thin PGMA brush film.

\section{Quantum Dot Nanoparticles}

We study PbS nanoparticle quantum dots, whose emission wavelengths are compatible with the Si photonic crystals, that is, the emission wavelength is longer than the Si band gap absorption edge (1100 nm). The nanoparticles were obtained from Suzhou Xingshuo Nanotech Co., Ltd. (MesoLight). From the peak of the emission spectrum, we estimated the average quantum dot diameter to be $D=3.47 \mathrm{~nm}$, using the calibration of Moreels et al. ${ }^{49}$ From the average diameter, we estimate that there are $N_{Q D}=402$ lead atoms per quantum dot. The inorganic lead sulfide core of the quantum dots is covered by a poly(ethylene glycol)-amine ligand that is used to couple to a PGMA polymer layer on the silicon photonic crystal.

\section{X-ray fluorescence tomogra- phy}

\section{X-ray fluorescence tomography setup}

Figure 6 shows the setup to perform X-ray fluorescence nano-tomography at ESRF beamline ID16A. 150151 X-ray fluorescence is excited by the incident beam with a photon energy set to $17 \mathrm{keV}$ with a $1 \%$ relative bandwidth. The X-ray beam is focused by multilayer coated Kirkpatrick-Baez (KB) optics onto the sample. At every sample orientation $\theta$, the exciting Xray focus is continuously scanned through the crystal in the horizontal direction and step-wise in the vertical direction to image the whole crystal. This corresponds to maps with $240 \times 240$ pixels (50 nm size), corresponding to a total

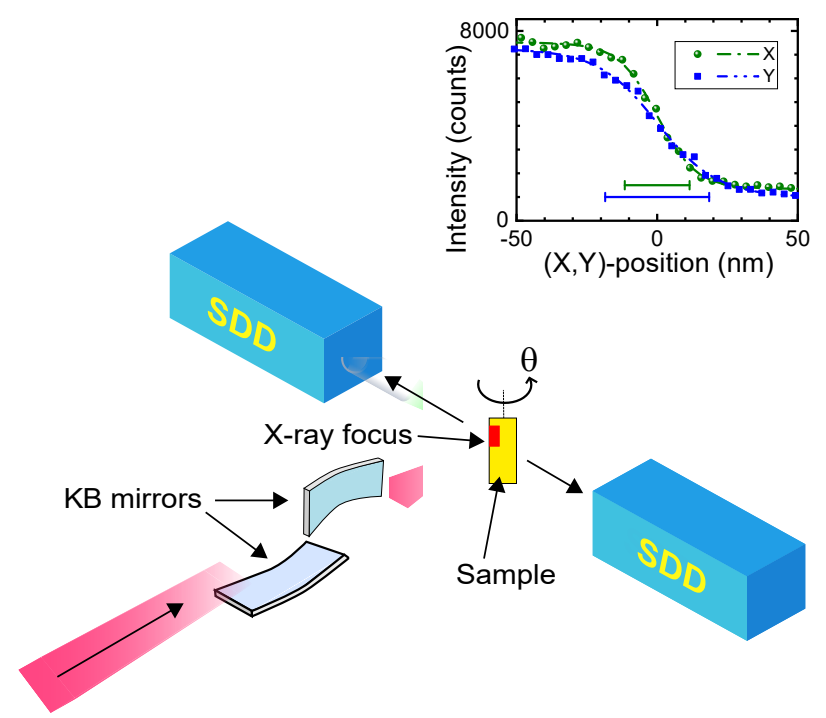

Figure 6: Schematic of the X-ray fluorescence setup. The X-ray beam arrives from bottom left, is focused by Kirkpatrick-Baez (KB) optics on the sample. The fluorescent light is detected by two silicon drift detectors (SDD) placed at $\pm 90^{\circ}$ with respect to the incident beam. The sample is rotated about the vertical axis (angle $\theta)$.

area of $12 \times 12 \mu \mathrm{m}^{2}$. At each sample position $\left(x_{i}, y_{j}\right)$ the $\mathrm{X}$-ray fluorescence photons are collected by two six-element silicon drift diode detectors that have an energy resolution of $130 \mathrm{eV}$ at $5 \mathrm{keV}$ photon energy and that are placed at $\pm 90^{\circ}$ with respect to the incident beam and that yield an energy-resolved spectrum $I\left(E, \theta, x_{i}, y_{j}\right)$. Each detection pixel has an area of $50 \times 50 \mathrm{~nm}^{2}$. The integration time at each sample position was set to $50 \mathrm{~ms}$. The flux of $7.5 \times 10^{9}$ photons/s is kept far below the maximum flux to prevent detector saturation, hence collecting one $240 \times 240$ pixel fluorescence image took about 1 hour, and collecting a full tomographic scan over 17 angles took about 20 hours, excluding the time for alignment.

\section{Volume reconstruction}

In the volume reconstruction the mass density distribution is reconstructed from the angular projections through tomographic reconstruction. In this case the Radon transform is severely undersampled and iterative, algebraic reconstruction methods have to be used. 
The volume reconstruction consists essentially of three steps: A) a drift correction on the projections, B) the tomographic reconstruction, C) corrections for self-absorption.

A) Drift correction: Due to the very long measurement times, lateral drifts occur that have to be corrected. The alignment was carried out with ESRF in-house software using the GNU Octave (http://www.octave.org) programming environment and the TomoJ plugin of the public domain image analysis program ImageJ (http://rsbweb.nih.gov/ij). The vertical and the horizontal drifts were separately corrected using the preservation of the vertical mass profile under rotation ${ }^{52}$ and the landmarks refinement of the TomoJ plugin, respectively. Gallium, the element that exactly defines the position of the pores, was used as the reference element for the drift correction. During the writing of the etch mask by focused-ion beam (FIB) the chromium hard mask is bombarded with gallium ions. That means that gallium is present on the surface at the locations where the pores are written. We used 4 landmarks on the elbow-shaped marker as suitable reference points. The data were corrected in such a way that the location of a specific landmark describes a sinusoidal trajectory for the different rotation angles. The drifts, as determined for gallium, were then compensated for on all elements using bicubic interpolation.

B) Volume reconstruction: Due to the small number of angles a regularized iterative tomographic reconstruction method was used. We used the the Total Variation Minimization (TVM) method with a positivity constraint as implemented in the PyHST software developed at ESRF. 53 The parameters for the regularization and the number of iterations were adjusted visually for the best outcome of the reconstruction. The final conditions of the reconstruction were as follows: 300 iterations of the Chambolle-Pock optimization algorithm without pre-conditioning and with positivity constraint and a TV regularization parameter of $10^{-5}$ and $10^{-3}$, respectively, for the elements $\mathrm{Ga}$ and $[\mathrm{Br}, \mathrm{Pb}]$. The sample substrate induces that the tomography is local in nature. To compensate for this the projections were padded horizontally by extension with the last value and the support of the volume reconstruction was taken to be a cylinder with 240 pixels height and 300 pixels diameter, i.e., slightly wider than the original projections.

C) Self absorption: To estimate the effect of the self absorption, we used the package $P y$ CorrectedEmissionCT. .54 Attenuations were included of both the incident $17 \mathrm{keV}$ X-ray beam and of the emitted X-ray fluorescence along its path to the detectors. We modelled the sample by a Si beam with 500 x $500 \mu \mathrm{m}^{2}$ crosssection and in one corner a $8 \times 8 \mu \mathrm{m}^{2}$ region with half the density of silicon representing the photonic crystal. For each element under study, a weighted average energy of the X-ray fluorescence was considered, and the corresponding attenuation coefficient was calculated using the Xraylib library. ${ }^{55}$ Next, attenuation maps per element were calculated for all angles of the tomography scan. As an illustration, Figure 7 shows the result for the $\mathrm{Pb}$ fluorescence at four angles, which show that the angle-dependent attenuation is fairly constant. Therefore, and to keep the tomographic inversion tractable, we chose to compensate for the self absorption by scaling-up all tomography by the inverse of the mean attenuation, averaged spatially over the photonic crystal and averaged over all tomography angles. This mean attenuation amounts to $0.168,0.404,0.415,0.445$ and 0.439 , respectively for $\mathrm{Cl}, \mathrm{Cu}, \mathrm{Ga}, \mathrm{Br}$ and $\mathrm{Pb}$.

\section{Acknowledgement}

We thank Matthijs Velsink, Diana Grishina, the MESA + Nanolab and ESRF staff for help. We are grateful to Nicola Viganò and Dmitry Karpov for their help in the self-absorption calculations with PyCorrectedEmissionCT. We acknowledge support by project "Tunable light sources by positioning quantum dots in 3D photonic bandgap crystals with polymer brushes" (712.012.003) of the "Nederlandse Organisatie voor Wetenschappelijk Onderzoek" (NWO), the $\mathrm{MESA}^{+}$Institute (Applied Nanophotonics, ANP), and the Descartes-Huygens Prize of the French Academy of Sciences to WLV (thanks to JMG). We thank ESRF for granting beamtime through experiment CH-5092. 


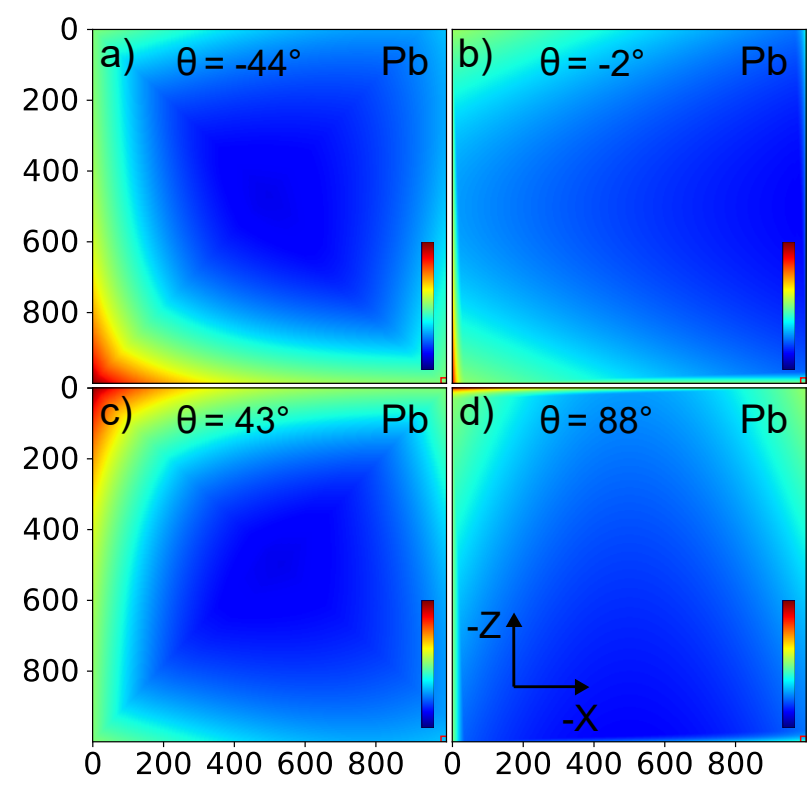

Figure 7: Attenuation maps of lead fluorescence as a function of atomic position inside the $\mathrm{Si}$ beam. In this representation, the Si-beam orientation is fixed with the photonic crystal (small square) at bottom right, whereas the incident X-ray beam orientation is varied: a) $\theta=-47^{\circ}$ beam incident from bottom left, $\mathrm{b}$ ) $\theta=-2^{\circ}$ beam from left, c) $\theta=43^{\circ}$ beam from top left, d) $\theta=88^{\circ}$ beam from top. All color bars run from 0 to 1 .

\section{Supporting Information Avail- able}

The Supporting Information is available free of charge on the ACS Publications website at DOI: 10.1021/acsnano.xxxx. The following information is available online: video animations showing:

S1 Scheme of the chemical synthesis.

S2 XZ cross-sections of Ga versus Y.

S3 XZ cross-sections of Br versus Y.

$\mathrm{S} 4 \mathrm{XZ}$ cross-sections of $\mathrm{Pb}$ versus $\mathrm{Y}$.

S5 Projections of Ga versus $\theta$.

S6 Projections of Br versus $\theta$.

S7 Attenuation for Ga for 4 angles.

S8 Attenuation for Br for 4 angles.
S9 Attenuation for $\mathrm{Cu}$ for 4 angles.

S10 Attenuation for $\mathrm{Cl}$ for 4 angles.

\section{References}

1. Novotny, L.; Hecht, B. Principles of NanoOptics; Cambridge University Press: Cambridge, 2006.

2. Tandaechanurat, A.; Ishida, S.; Guimard, D.; Nomura, M.; Iwamoto, S.; Arakawa, Y. Lasing Oscillation in a ThreeDimensional Photonic Crystal Nanocavity with a Complete Bandgap. Nature Photon. 2011, 5, 91-94.

3. Leistikow, M. D.; Mosk, A. P.; Yeganegi, E.; Huisman, S. R.; Lagendijk, A.; Vos, W. L. Inhibited Spontaneous Emission of Quantum Dots Observed in a 3D Photonic Band Gap. Phys. Rev. Lett. 2011, 10\%, 193903: 1-5.

4. Atwater, H. A.; Polman, A. Plasmonics for Improved Photovoltaic Devices. Nature Mater. 2010, 9, 205-213.

5. Battaglia, C.; Hsu, C.; Soderstrom, K.; Escarre, J.; Haug, F.; Charriere, M.; Boccard, M.; Despeisse, M.; Alexander, D. T. L.; Cantoni, M.; Cui, Y.; Ballif, C. Light Trapping in Solar Cells: Can Periodic Beat Random? ACS Nano 2012, 6, 2790-2797.

6. Gu, W.; Yushin, G. Review of nanostructured carbon materials for electrochemical capacitor applications: advantages and limitations of activated carbon, carbidederived carbon, zeolite-templated carbon, carbon aerogels, carbon nanotubes, onionlike carbon, and graphene. WIREs Energy and Environment 2014, 3, 424-473.

7. Sun, Y.-F.; Liu, S.-B.; Meng, F.-L.; Liu, J.Y.; Jin, Z.; Kong, L.-T.; Liu, J.-H. Metal Oxide Nanostructures and Their Gas Sensing Properties: A Review. Sensors 2012, 12, 2610-2631. 
8. Zhang, X.; Cheng, X.; Zhang, Q. Nanostructured energy materials for electrochemical energy conversion and storage: A review. Journal of Energy Chemistry 2016, 25, $967-984$.

9. Wang, Y.; Cao, G. Developments in Nanostructured Cathode Materials for HighPerformance Lithium-Ion Batteries. Advanced Materials 2008, 20, 2251-2269.

10. Ji, L.; Lin, Z.; Alcoutlabi, M.; Zhang, X. Recent developments in nanostructured anode materials for rechargeable lithium-ion batteries. Energy Environ. Sci. 2011, 4, 2682-2699.

11. Goriparti, S.; Miele, E.; De Angelis, F.; Di Fabrizio, E.; Proietti Zaccaria, R.; Capiglia, C. Review on recent progress of nanostructured anode materials for Li-ion batteries. Journal of Power Sources 2014, $257,421-443$.

12. Li, F.; Zhou, Z. Micro/Nanostructured Materials for Sodium Ion Batteries and Capacitors. Small 2018, 14, 1702961.

13. Koenderink, A. F.; Bechger, L.; Schriemer, H. P.; Lagendijk, A.; Vos, W. L. Broadband Fivefold Reduction of Vacuum Fluctuations Probed by Dyes in Photonic Crystals. Phys. Rev. Lett. 2002, 88, 143903.

14. Andrew, P.; Barnes, W. L. Energy Transfer Across a Metal Film Mediated by Surface Plasmon Polaritons. Science 2004, 306, 1002-1005.

15. Taminiau, T. H.; Stefani, F. D.; Segerink, F. B.; van Hulst, N. F. Optical antennas direct single-molecule emission. Nature Photonics 2008, 2, 234-237.

16. Nozik, A. J.; Beard, M. C.; Luther, J. M.; Law, M.; Ellingson, R. J.; Johnson, J. C. Semiconductor Quantum Dots and Quantum Dot Arrays and Applications of
Multiple Exciton Generation to ThirdGeneration Photovoltaic Solar Cells. Chemical Reviews 2010, 110, 6873-6890, PMID: 20945911.

17. Rühle, S.; Shalom, M.; Zaban, A. Quantum-Dot-Sensitized Solar Cells. ChemPhysChem 2010, 11, 2290-2304.

18. Gérard, J. M.; Sermage, B.; Gayral, B.; Legrand, B.; Costard, E.; Thierry-Mieg, V. Enhanced Spontaneous Emission by Quantum Boxes in a Monolithic Optical Microcavity. Phys. Rev. Lett. 1998, 81, 11101113

19. Anger, P.; Bharadwaj, P.; Novotny, L. Enhancement and Quenching of SingleMolecule Fluorescence. Phys. Rev. Lett. 2006, 96, 113002.

20. Barnes, W. L.; Horsley, S. A. R.; Vos, W. L. Classical antennae, quantum emitters, and densities of optical states. Journal of Optics 2020 ,

21. Vats, N.; John, S.; Busch, K. Theory of Fluorescence in Photonic Crystals. Phys. Rev. A 2002, 65, 043808.

22. Mavidis, C. P.; Tasolamprou, A. C.; Hasan, S. B.; Koschny, T.; Economou, E. N.; Kafesaki, M.; Soukoulis, C. M.; Vos, W. L. Local density of optical states in the three-dimensional band gap of a finite photonic crystal. Phys. Rev. B 2020, 101, 235309.

23. Zhu, C.; Yang, G.; Li, H.; Du, D.; Lin, Y. Electrochemical Sensors and Biosensors Based on Nanomaterials and Nanostructures. Analytical Chemistry 2015, 87, 230249, PMID: 25354297.

24. Ho, K. M.; Chan, C. T.; Soukoulis, C. M.; Biswas, R.; Sigalas, M. Photonic band gaps in three dimensions: New layer-by-layer periodic structures. Solid State Commun. 1994, 89, 413-416.

25. Matyjaszewski, K. Atom Transfer Radical Polymerization (ATRP): Current Status 
and Future Perspectives. Macromolecules 2012, 45, 4015-4039.

26. Schulz, A. S.; Gojzewski, H.; Huskens, J.; Vos, W. L.; Vancso, G. J. Controlled sub-10-nanometer poly(N-isopropylacrylamide) layers grafted from silicon by atom transfer radical polymerization. Polym. Adv. Technol. 2018, 29, 806-813.

27. Goldstein, J. I.; Newbury, D. E.; Michael, J. R.; Ritchie, N. W.; Scott, J. H. J.; Joy, D. C. Scanning electron microscopy and X-ray microanalysis; Springer, 2017.

28. Belu, A. M.; Graham, D. J.; Castner, D. G. Time-of-flight secondary ion mass spectrometry: techniques and applications for the characterization of biomaterial surfaces. Biomaterials 2003, 24, 3635-3653.

29. Chastain, J.; King Jr, R. C. Handbook of X-ray photoelectron spectroscopy. PerkinElmer, USA 1992, 261.

30. Demtröder, W. Laser Spectroscopy 2; Springer Berlin Heidelberg, 2015.

31. Misra, S.; Liu, N.; Nelson, J.; Hong, S. S.; Cui, Y.; Toney, M. In Situ X-Ray Diffraction Studies of (De)Lithiation Mechanism in Silicon Nanowire Anodes. ACS Nano 2012, 9, 5465-5473.

32. Stankevič, T.; Hilner, E.; Seiboth, F.; Ciechonski, R.; Vescovi, G.; Kryliouk, O.; Johansson, U.; Samuelson, L.; Wellenreuther, G.; Falkenberg, G.; Feidenhans'l, R.; Mikkelsen, A. Fast Strain Mapping of Nanowire Light-Emitting Diodes Using Nanofocused X-Ray Beams. ACS Nano 2015, 9, 6978-6984.

33. Haubold, H.; Gruenhagen, K.; Wagener, M.; Jungbluth, H.; Heer, H.; Pfeil, A.; Rongen, H.; Brandenberg, G.; Moeller, R.; Matzerath, J.; Hiller, P.; Halling, H. JUSIFA - A new user-dedicated ASAXS beamline for materials science. $R e$ view of Scientific Instruments 1989, 60, 1943-1946.
34. Seifert, S.; Winans, R.; Tiede, D.; Thiyagarajan, P. Design and performance of a ASAXS instrument at the Advanced Photon Source. Journal of Applied Crystallography 2000, 33, 782-784.

35. Bleuet, P.; Simionovici, A.; Lemelle, L.; Ferroir, T.; Cloetens, P.; Tucoulou, R.; Susini, J. Hard x-rays nanoscale fluorescence imaging of Earth and Planetary science samples. Applied Physics Letters 2008, 92, 213111.

36. Silversmit, G.; Vekemans, B.; Brenker, F. E.; Schmitz, S.; Burghammer, M.; Riekel, C.; Vincze, L. X-ray Fluorescence Nanotomography on Cometary Matter from Comet 81P/Wild2 Returned by Stardust. Analytical Chemistry 2009, 81, 6107-6112, PMID: 19572556.

37. de Jonge, M. D.; Vogt, S. Hard X-ray fluorescence tomography - an emerging tool for structural visualization. Curr. Opin. Struct. Biology 2010, 20, 606-614.

38. Woldering, L. A.; Tjerkstra, R. W.; Jansen, H. V.; Setija, I. D.; Vos, W. L. Periodic arrays of deep nanopores made in silicon with reactive ion etching and deep UV lithography. Nanotechnology 2008, 19, 145304 .

39. Grishina, D. A.; Harteveld, C. A. M.; Woldering, L. A.; Vos, W. L. Method for making a single-step etch mask for 3D monolithic nanostructures. Nanotechnology 2015, 26, 505302 .

40. Grishina, D. A.; Harteveld, C. A. M.; Pacureanu, A.; Devashish, D.; Lagendijk, A.; Cloetens, P.; Vos, W. L. X-ray Imaging of Functional Three-Dimensional Nanostructures on Massive Substrates. ACS Nano 2019, 13, 13932-13939, PMID: 31829557.

41. Vincze, L.; Vekemans, B.; Brenker, F. E.; Falkenberg, G.; Rickers, K.; Somogyi, A.; Kersten, M.; Adams, F. Three-Dimensional 
Trace Element Analysis by Confocal Xray Microfluorescence Imaging. Analytical Chemistry 2004, 76, 6786-6791, PMID: 15538804 .

42. Alonso-Mori, R.; Kern, J.; Sokaras, D.; Weng, T.-C.; Nordlund, D.; Tran, R.; Montanez, P.; Delor, J.; Yachandra, V. K.; Yano, J.; Bergmann, U. A multi-crystal wavelength dispersive x-ray spectrometer. Review of Scientific Instruments 2012, 83, 073114 .

43. Zhang, J.-N.; Li, Q.; Ouyang, C.; Yu, X.; Ge, M.; Huang, X.; Hu, E.; Ma, C.; Li, S.; Xiao, R.; Yang, W.; Chu, Y.; Liu, Y.; Yu, H.; Yang, X.-Q.; Huang, X.; Chen, L.; $\mathrm{Li}, \mathrm{H}$. Trace doping of multiple elements enables stable battery cycling of LiCoO2 at 4.6 V. Nature Energy 2019, 4, 594-603.

44. van den Broek, J. M.; Woldering, L. A.; Tjerkstra, R. W.; Segerink, F. B.; Setija, I. D.; Vos, W. L. Inverse-Woodpile Photonic Band Gap Crystals with a Cubic Diamond-like Structure Made from SingleCrystalline Silicon. Adv. Funct. Mater. 2012, 22, 25-31.

45. Woldering, L. A.; Mosk, A. P.; Tjerkstra, R. W.; Vos, W. L. The influence of fabrication deviations on the photonic band gap of three-dimensional inverse woodpile nanostructures. J. Appl. Phys. 2009, 105, 093108.

46. Kern, W. The Evolution of Silicon Wafer Cleaning Technology. J. Electrochem. Soc. 1990, 137, 1887-1892.

47. Ramakrishnan, A.; Dhamodharan, R.; Rühe, J. Controlled growth of PMMA brushes on silicon surfaces at room temperature. Macromol. Rapid Commun. 2002, 23, 612-616.

48. Edmondson, S.; Huck, W. T. S. Controlled growth and subsequent chemical modification of poly(glycidyl methacrylate) brushes on silicon wafers. J. Mater. Chem. 2004, $14,730-734$.
49. Moreels, I.; Lambert, K.; Smeets, D.; De Muynck, D.; Nollet, T.; Martins, J. C.; Vanhaecke, F.; Vantomme, A.; Delerue, C.; Allan, G.; Hens, Z. Size-Dependent Optical Properties of Colloidal PbS Quantum Dots. ACS Nano 2009, 3, 3023-3030, PMID: 19780530.

50. da Silva, J. C.; Pacureanu, A.; Bohic, S.; Morawe, C.; Barrett, R.; Cloetens, Efficient Concentration of High-Energy XRays for Diffraction-Limited Imaging Resolution. Optica 2017, 4, 492-495.

51. Villar, F.; Andre, L.; Baker, R.; Bohic, S.; da Silva, J. C.; Guilloud, C.; Hignette, O.; Meyer, J.; Pacureanu, A.; Perez, M.; Salome, M.; van der Linden, P.; Yang, Y.; Cloetens, P. Nanopositioning for the ESRF ID16A Nano-Imaging Beamline. Synchrotron Radiat. News 2018, 31, 9-14.

52. Guizar-Sicairos, M.; Diaz, A.; Holler, M.; Lucas, M. S.; Menzel, A.; Wepf, R. A.; Bunk, O. Phase tomography from x-ray coherent diffractive imaging projections. Opt. Express 2011, 19, 21345-21357.

53. Mirone, A.; Brun, E.; Gouillart, E.; Tafforeau, P.; Kieffer, J. The PyHST2 hybrid distributed code for high speed tomographic reconstruction with iterative reconstruction and a priori knowledge capabilities. Nuclear Instruments and Methods in Physics Research Section B: Beam Interactions with Materials and Atoms 2014, 324, 41-48, 1st International Conference on Tomography of Materials and Structures.

54. Viganò, N. R.; Solé, V. A. Physically corrected forward operators for induced emission tomography: a simulation study. $\mathrm{Mea}$ surement Science and Technology 2018, 29, 034005 .

55. Schoonjans, T.; Brunetti, A.; Golosio, B.; Sanchez del Rio, M.; Solé, V. A.; Ferrero, C.; Vincze, L. The xraylib library for $\mathrm{X}$-ray-matter interactions. Recent developments. Spectrochimica Acta Part B: Atomic Spectroscopy 2011, 66, 776-784. 


\section{Graphical TOC Entry}

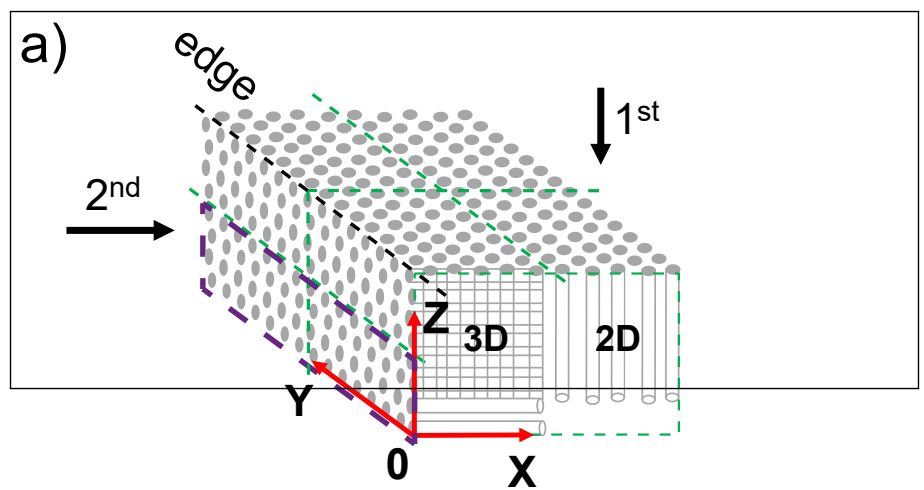

b)

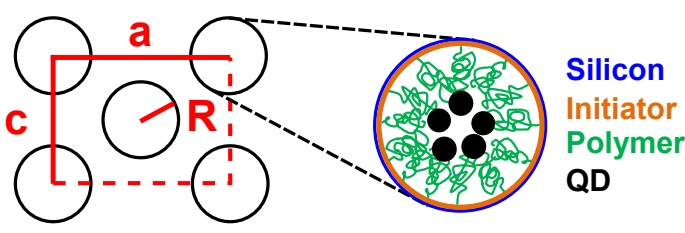

(a) Schematic of an inverse woodpile photonic crystal on the edge of a silicon beam. Green dashed lines indicate the two surfaces into which nanopores are etched. The 3D and 2D parts of the crystal are indicated in the XZ front face. (b) View along the pores showing the lattice parameters $a, c$ (with $a=\sqrt{2} c$ ) and pore radius $\mathrm{R}$. Zoomed-in cross-section of one pore with targeted surface-chemistry: ATRP initiator layer (orange), polymer chains forming brushes (green) and covalently attached $\mathrm{PbS}$ quantum dots (red) on top of silicon (black). 